\title{
SOME NEW OSTROWSKI-TYPE BOUNDS FOR THE ČEBYŠEV FUNCTIONAL AND APPLICATIONS
}

\author{
P. CERone AND S. S. Dragomir
}

Abstract. Some new inequalities of Ostowski-type for the Čebyšev functional and applications for Taylor's expansion and generalised trapezoid formula are pointed out.

Mathematics subject classification (2010): Primary 26D15; Secondary 41A55.

Keywords and phrases: Čebyšev functional, Grüss inequality, Čebyšev inequality, Ostrowski inequality, generalised trapezoid formula, Taylor's expansion, Lupaş's inequality.

\section{REFERENCES}

[1] G. A. Anastassiou And S. S. DRAgOmir, On some estimates of the remainder in Taylor's formula, Journal of Mathematical Analysis and Applications 263 (2001), 246-263.

[2] P. Cerone, S. S. Dragomir, J. Roumeliotis AND J. S̆Unde, A new generalisation of the trapezoid formula for $n$-time differentiable mappings and applications, Demonstratio Math. 33, 4 (2000), 719-736.

[3] P. L. Chebyshev, Sue les expressions approximatives des intégrales définies par les autres prises entre les mêmes limites, Proc. Math. Soc. Charkov 2 (1982), 93-98.

[4] G. GRÜSs, Über das Maximum des absoluten Betrages von $\frac{1}{b-a} \int_{a}^{b} f(x) g(x) d x-\frac{1}{(b-a)^{2}} \int_{a}^{b} f(x) d x$ $\int_{a}^{b} g(x) d x$, Math. Z. 39 (1934), 215-226.

[5] S.-R. HwANG, Ostrowski-Grüss-Cebyšev type inequalities involving several functions, Tamsui Oxf. J. Math. Sci. 23, 1 (2007), 105-125.

[6] Z. LiU, Generalizations of some new Čebyšev type inequalities, J. Inequal. Pure Appl. Math. 8, 1 (2007), Article 13, 6 pp. (electronic).

[7] A. LuPAș, The best constant in an integral inequality, Mathematica (Cluj) 15, 38 (1973), 219-222.

[8] M. MatiĆ, J. PeČARIĆ AND N. UjeVIĆ, On new estimation of the remainder in generalised Taylor's formula, Math. Ineq. \& Appl. 2, 3 (1999), 343-361.

[9] D. S. Mitrinović, J. PeČArić And A. M. Fink, Classical and New Inequalities in Analysis, Kluwer Academic Publishers, 1993.

[10] B. Mond, J. PeČARIĆ AND B. TePeš, Counterparts of Schwarz's inequality for Čebyšev functional, J. Appl. Funct. Anal. 1, 1 (2006), 57-66.

[11] A. M. Ostrowski, On an integral inequality, Aequat. Math. 4 (1970), 358-373.

[12] B. G. PAChPATte, On Ostrowski-Grüss-Čebyšev type inequalities for functions whose modulus of derivatives are convex, J. Inequal. Pure Appl. Math. 6, 4 (2005), Article 128, 15 pp. (electronic).

[13] B. G. PAChPATte, On Čebyšev-Grüss type inequalities via Pečarić's extension of the Montgomery identity, J. Inequal. Pure Appl. Math. 7, 1 (2006), Article 11, 4 pp. (electronic).

[14] B. G. PAChPATte, New Čebyšev type inequalities via trapezoidal-like rules, J. Inequal. Pure Appl. Math. 7, 1 (2006), Article 31, 6 pp. (electronic).

[15] B. G. PachPatte, A note on Čebyšev type inequalities, An. Ştiinţ. Univ. Al. I. Cuza Iaşi. Mat. (N.S.) 53, 1 (2007), 97-102.

[16] B. G. PachPatte, New Čebyšev like integral inequalities, Advances in inequalities from probability theory and statistics, 155-161, Adv. Math. Inequal. Ser., Nova Sci. Publ., New York, 2008.

[17] A. Rafic, Q. Shahbaz And A. M. Acu, The generalized Čebyšev type inequality, Sci. Stud. Res. Ser. Math. Inform. 19, 1 (2009), 195-200. 
[18] E. Set, M. Z. SARIKAYA AND F. AhmAd, A generalization of Čebyšev type inequalities for first differentiable mappings, Miskolc Math. Notes 12, 2 (2011), 245-253.

[19] F. ZADAR AND N. A. MIR, A note on the generalization of some new Čebyšev type inequalities, Tamsui Oxf. J. Inf. Math. Sci. 27, 2 (2011), 149-157. 